vendre pendant plusieurs mois au même titre que les limonades ou les jus de fruits et que l'on peut ainsi stocker.

Il en est de même pour les crèmes et les laits condensés que l'on peut conserver dans des récipients en verre. Pour le lait de consommation journalière, les applications du procédé sont encore limitées. Néanmoins, il est prou vé que l'emploi du soutirage stérile, par une simplification du procédé, - des essais pratiques sont actuellement en cours - permet de prolonger sensiblement la conservation du lait en bouteilles en général tout en lui maintenant ses qualités. On peut atteindre aujourd'hui une conservation de 3 à 4 jours.

Il faut espérer que dans un avenir proche des essais seront entrepris pour résoudre en détail le problème de l'aug mentation de la durée de conservation, indépendamment de la stérilisation absolue et du soutirage stérile.

Cette question ouvrira sans aucun doute de nombreux débouchés à l'industrie laitière. Que l'on réussise tout d'abord à produire du lait en bouteilles se différenciant à peine du lait pasteurisé et ayant une durée de conservation de plusieurs jours, il est incontestable que ceci aura naturellement une influence sur le mode de travail des entreprises et sur le prix de revient, non seulement, en raison du fait que le consommateur recevra son lait en bouteilles sous une forme meilleure qui, grâce à la conservation, évitera les pertes, mais aussi parce qu'on pourra ainsi réduire les frais de l'entreprise, ce qui aura pour résultat final d'ouvrir de nouveaux débouchés à l'industrie du lait en bouteilles présenté de cette manière essentiellement hygiénique.

\title{
SÉPARATION DU LACTOSE ET DES PROTÉINES SOLUBLES CONTENUES DANS LE SÉRUM DU LAIT PAR EXTRACTION PAR L'ALCOOL
}

par G. GÉNIN

Ingénieur E. P. C.

On sait déjà depuis longtemps, à la suite d'études expérimentales entreprises sur la question, que la poudre de petit-lait ou de sérum provenant de la fabrication de la caséine peut être extraite au moyen de solutions alcooliques. On a constaté, en opérant avec des solutions alcooliques de différentes concentrations, qu'il existe, au voisinage d'une concentration en alcool de $70,7 \%$, une zone étroite au-dessus de laquelle la précipitation par l'alcool des constituants de la poudre n'entraîne pas de modification, de la solubilité des protéines du sérum dans l'eau, mais au-dessous de laquelle cette solubilité est diminuée. 
Ces recherches expérimentales ont montré également que le lactose qui existe dans la poudre de petit-lait est présent sous une forme amorphe ou vitreuse; dans ces conditions, le premier effet qu'exerce sur le lactose l'addition d'alcool à la poudre, à côté de réaliser un changement de solvant, est de produire une solution sursaturée de lactose. La stabilité de cette solution sursaturée est telle qu'une extraction complète du lactose et sa séparation des substances insolubles par filtration est possible. On fait alors cristalliser le lactose contenu dans le filtrat et on le recueille.

Ces constatations que nous venons brièvement de résumer, ainsi que d'autres faits, sont à la base d'un procédé d'extraction [1] des constituants de la poudre de sérum que l'on peut isoler de la façon suivante :

a) Un lactose d'excellente qualité que l'on obtient sous forme cristallisée avec un rendement de $80 \%$.

b) Une poudre soluble contenant environ $50 \%$ de protéines, exempte de lactose et ne présentant aucun goût. Cette poudre contient également $15 \%$ de cendres et $35 \%$ de matières solides mais qui sont volatiles à la température de calcination.

c) Un produit alimentaire riche en lactoflavine.

Dans l'ancien procédé de préparation du lactose en partant du sérum, on suivait une technique qui consistait à régler les conditions de l'opération et la température, de telle sorte qu'il y ait coagulation de la majeure partie des protéines et précipitation d'une partie des sels. On séparait alors le précipité par décantation et filtration; le filtrat était concentré dans le vide jusqu'à cristallisation du lactose. Après séparation, les cristaux étaient purifiés par une seconde cristallisation. On récupérait de cette façon environ $50 \%$ du lactose contenu dans le sérum, mais les protéines étaient dénaturées par la chaleur et devenaient insolubles.

On a alors réalisé de nouveaux progrès en permettant, par un réglage de la réaction du milieu et de la température, au sucre de cristalliser sans qu'il soit nécessaire de séparer les protéines et de les rendre insolubles [2]. En opérant dans ces conditions, on obtient une liqueur, qui, après séparation des cristaux de sucre, peut être desséchée et transformée en poudre, cette dernière étant désignée sous le nom de poudre de protéine de sérum; elle contient encore 37 à $52 \%$ de lactose, 3 à $45 \%$ de protéines et 12 à $18 \%$ de cendres. Certains sels peuvent d'ailleurs être éliminés de la poudre par dialyse [3], ce qui augmente la proportion relative de lactose et de protéines.

C'est alors qu'on a songé à appliquer industriellement le procédé 
d'extraction par l'alcool de façon à réaliser une technique répondant aux conditions suivantes :

a) Obtenir un meilleur rendement en lactose par rapport à ce produit contenu dans la poudre initiale.

b) Obtenir, en une seule cristallisation, un lactose de grande pureté.

c) Obtenir des protéines pratiquement exemptes de lactose et de sels, sans que leurs propriétés soient réduites et en particulier sans réduction de leur solubilité et de leur pouvoir de donner des solutions formant des mousses abondantes par battage.

Les travaux qui ont conduit à la mise au point de ce procédé ont fait l'objet d'une communication récente dont nous soulignerons les points essentiels [4]. Disons tout de suite que le nouveau procédé qui, comme nous le disions plus haut, repose sur l'emploi de l'alcool comme agent d'extraction, n'est pas seulement applicable à la poudre de petit-lait obtenue par atomisation, mais également aux poudres de lait écrémé ou de protéines du sérum, au lait écrémé concentré et aux solutions concentrées de protéines du sérum.

Nous ne décrirons pas les recherches de LEviton et LEIGHTON qui ont porté sur la mise au point d'un appareillage d'extraction de méthodes analytiques et qui ont permis également de mettre en évidence les points suivants : lait.

Pouvoir solvant de l'alcool à $70,7 \%$ sur la poudre de petit-

Influence des variations de la quantité de solvant.

Variation de la pureté du lactose en fonction du $p \mathrm{H}$ du filtrat.

Influence de la concentration de l'alcool sur la solubilité des protéines du petit-lait.

Nous nous contenterons de donner deux exemples qui permettent, par des essais de laboratoire, de se rendre compte de l'intérêt de cette nouvelle méthode d'extraction du lactose.

Exemple 1. - Cet exemple a pour but de montrer l'intérêt de l'emploi de l'alcool à 70,7\% comme agent d'extraction du lactose. On est parti de 100 grammes de poudre de petit-lait doux, séché par atomisation, et contenant $69,1 \%$ de lactose et $12,5 \%$ de matières azotées calculées en albumine. Cette poudre est agitée rapidement à la température ordinaire de $25^{\circ}$ avec environ $300 \mathrm{~cm}^{3}$ d'alcool à $95 \%$. L'opération ne doit pas demander plus de temps qu'il n'en faut pour obtenir un mélange homogène. On ajoute alors à ce mélange un autre mélange d'alcool et d'eau en quantités telles que, 
dans le produit final, deux litres de solvant contiennent des volumes d'alcool à 95 et d'eau dans le rapport 4 à 1 . Le mélange est alors agité pendant quelques minutes, puis filtré rapidement.

Le filtrat contient tout le lactose en solution, et est acidifié par addition de $1 \%$ en volume d'acide chlorhydrique, $1,7 \mathrm{~N}$ en solution dans l'alcool absolu ; dans ces conditions le $p H$ du filtrat est abaissé de 5,38 à 2,75. Après 15 heures d'agitation $80 \%$ du lactose contenu dans la poudre initiale peuvent être recueillis sous la forme cristallisée. Ces cristaux sont alors mélangés avec 4 fois leur poids d'alcool à $70,7 \%$ en vue d'un bon lavage ; on obtient finalement un lactose qui ne contient que $0,011 \%$ d'azote et $0,14 \%$ de cendres formées surtout de carbonates. Lorsque ce lactose est dissous dans la concentration de $5 \%$, il donne des solutions limpides et neutres au papier de tournesol.

Le résidu insoluble dans le solvant et séparé par la filtration est lavé avant de le sécher à la température ordinaire avec une quantité suffisante d'alcool pour rendre la concentration de la liqueur contenue dans le précipité égale ou supérieure à la concentration d'un mélange azéotropique. On recueille ainsi 15 gr. 6 de résidu. Ce dernier ne contient pas de quantité appréciable de lactose, il renferme $47,8 \%$ de protéines et $16 \%$ de cendres.

L'examen de ce résidu a montré que $15,8 \%$ des matières solides qu'il contient sont formées de substances insolubles ou en suspension colloïdale, les produits les plus grossièrement dispersés comprennent principalement des sels insolubles. Lorsqu'on prépare en partant du résidu une suspension à $10 \%$ dans l'eau, cette suspension mousse abondamment par battage et ne présente aucun goût salé.

Exemple 2. - Cet exemple, contrairement au précédent, a pour but de montrer l'emploi de l'alcool à $95 \%$ comme solvant. On part d'une partie de poudre de petit-lait que l'on agite avec 80 parties d'alcool à $95 \%$ en opérant à la température de $60^{\circ} \mathrm{C}$. Au bout d'une minute, la suspension obtenue par cette agitation est filtrée. Le filtrat contient par $100 \mathrm{~cm}^{3} 0$ gr. 465 de lactose, 0 gr. 027 de cendres, 0 gr. 020 de protéines et 0 gr. 568 de matières solides totales. La quantité de lactose contenue dans la totalité du filtrat représente $68 \%$ du lactose contenu initialement dans la poudre.

Ce filtrat est alors acidifié par $0,25 \%$ en volume d'acide chlorhydrique, 1,7 N et refroidit rapidement à la température ordinaire. Au bout de quelques heures, le lactose, qui a cristallisé, est séparé et lavé avec de l'alcool à $95 \%$, on obtient ainsi un rendement en lactose de $60 \%$. L'analyse du sucre ainsi recueilli montre qu'il contient $0,005 \%$ d'azote et $0,09 \%$ de cendres. Il donne des 
solutions aqueuses limpides et l'analyse polarimétrique montre qu'il contient 1,3 partie de $\beta$-lactose pour 1 partie d' $\alpha$-lactose.

Quand on part de 115 grammes de poudre de petit-lait, on obtient 57 grammes d'un résidu qui contient $25 \%$ de protéines, $14,2 \%$ de cendres et $52,5 \%$ de lactose. Les suspensions aqueuses de ce résidu moussent facilement et possèdent un goût sucré agréable.

Avec quelques modifications de détail, ce procédé d'extraction par l'alcool peut être appliqué au traitement de la poudre de petitlait sur et au petit-lait concentré.

Au point de vue industriel, ce procédé pose un problème de récupération de solvants, en l'occurrence d'alcool, mais cette récupération ne comporte qu'une simple distillation d'alcool; ce n'est que dans le cas du petit-lait concentré que la distillation doit être accompagnée d'une rectification effectuée au moyen d'une colonne à rectifier. Lorsque l'on part d'ailleurs de petit-lait concentré, il peut être avantageux de récupérer une partie du lactose par la méthode de Bell, Peter et Johnson [5]. D'après cette méthode, le petit-lait est concentré jusqu'à ce que la solution soit fortement sursaturée, en ce qui concerne le lactose, ce sucre cristallise et on le sépare. Toutefois il reste encore dans la solution, sous forme dissoute, une quantité relativement importante de lactose représentant 37 à $52 \%$ des matières solides. C'est sur cette solution résiduaire que l'on procède alors à l'extraction par l'alcool.

Le procédé d'extraction par l'alcool est également applicable à la préparation du lactose en partant de lait écrémé concentré ou de poudre de lait écrémé. Toutefois dans ce cas la caséine contenue dans ces produits de départ est dénaturée même lorsqu'on emploie des solutions alcooliques à haute teneur en alcool.

On notera enfin que la liqueur-mère neutralisée contient encore, après séparation du lactose et élimination de l'alcool, une certaine quantité de substances solides dont $58 \%$ de lactose, $10 \%$ de matières azotées et $21,5 \%$ de cendres. De plus, ees substances solides se sont considérablement enrichies en lactoflavine. Les protéines contenues dans ces liqueurs-mères ne sont pratiquement pas coagulées par la chaleur et sont probablement du type soluble dans l'alcool signalé par OsBorne et WAKEMAN [6].

Dans le procédé d'extraction par l'alcool que nous avons décrit, l'emploi comme solvant d'alcool à $95 \%$ présente un intérêt exceptionnel, ear il permet d'obtenir un lactose extraordinairement pur, sucré et soluble. Ce lactose est constitué par un mélange finement divisé de $\alpha$ et de $\beta$-lactose dans les proportions correspondant à celles qui existent dans une solution équilibrée de lactose. Mais malheureusement lorsqu'on emploie un alcool de cette concentration, il faut utiliser 10 litres d'alcool pour traiter seulement $100 \mathrm{gr}$. 
de poudre de sérum, ce qui rend le procédé difficilement applicable industriellement. On notera néanmoins que le rendement en lactose après 2 heures d'extraction seulement est déjà satisfaisant et que le temps nécessaire pour produire la cristallisation du lactose peut être sensiblement réduit par agitation; dans ces conditions, les opérations sont plus rapides, les conditions d'utilisation du solvant sont plus favorables qu'arec l'alcool à $70,7 \%$, puisque nous avons $v u$ que dans ce dernier cas une durée de cristallisation de 15 heures est nécessaire pour obtenir un rendement satisfaisant.

La poudre de protéines que l'on obtient comme sous-produit de l'extraction du lactose contient environ $50 \%$ de protéi nes. Le prétraitement $\mathrm{du}$ petit-lait pour éliminer les substances insolubles : caséine et sels de calcium insolubles, améliore considérablement la qualité des produits que l'on obtient par extraction par l'alcool : lactose et poudre de protéine. En particulier, la proportion d'impuretés insolubles devient insignifiante.

Néanmoins, malgré cette purification préliminaire, la poudre dite poudre de protéines ne eontient pas plus de $50 \%$ de protéines, elle renferme en outre $15 \%$ de cendres et le reste est donc constitué de substances détruites au cours de la calcination, probablement des lactases et des citrates solubles et insolubles. Il faudrait, pour augmenter la proportion de protéines dans la poudre, pouvoir traiter le sérum de départ pour éliminer le citrate et le lactate de calcium.

\section{RÉFÉRENCES}

[1] A. Leviton. Demande de brevet déposée aux Etats-Unis, non encore publiée.

[2] Beli, Peter et Johnson. Journal Dairy Sci., t. IJ, 1928, p. 163.

[3] P. D. WAtson. Md. Eng. Chem., t. XXVI, 1934, p. 640.

[4] A. Leviton et A. Leighton. Md. Eng. Chem., t. XXX, 1938, p. 1305.

[5] Bell, Peter et Johnson. Loc. eit.

[6] T. B. Osborne et A. J. Wakeman, Journal Biol. Chem., t. XXXIII, 1918, p. 243.

\section{REVUES}

LES FROMAGES A LA GRÈME (I)

TEGHNIQUE DE FABRICATION DE QUELQUES TYPES DE GES FROMAGES.

\section{Fromage double-crème}

Ce fromage de fabrication assez facile donne un produit de toute première qualité. Il constitue un intermédiaire entre le double-

(1) D'après le Dr J. M. Rosell, professeur à l'Ecole de Laiterie de la province de Québec. 